\title{
Sonographic Evaluation of the Median Nerve at the Wrist
}

\author{
David A. Jamadar, MB, BS, Jon A. Jacobson, $M D$, \\ Curtis W. Hayes, MD
}

Received March 23, 2001, from the Department of Radiology, University of Michigan Hospitals, Ann Arbor, Michigan. Revision requested April 30, 2001. Revised manuscript accepted for publication June 4, 2001.

Address correspondence and reprint requests to David $A$. Jamadar, MB, BS, Radiology, TC 2910, University of Michigan Hospitals, 1500 E Medical Center Dr, Ann Arbor, MI 48109-0326.

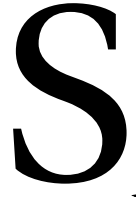
onography is now used to evaluate many disorders of the musculoskeletal system. ${ }^{1}$ One such example is evaluation of the median nerve in carpal tunnel syndrome. ${ }^{2}$ Sonographic findings such as median nerve enlargement immediately proximal to the carpal tunnel and bowing of the flexor retinaculum have been described in these patients. ${ }^{2}$ A correct diagnosis relies on accurate identification of the median nerve and an understanding of the osseous landmarks delineating the carpal tunnel.

The presence of multiple closely approximated linear echogenic structures at the volar wrist makes identification of the median nerve difficult; differentiation from the palmaris longus tendon and other tendons may occasionally prove challenging. We present a technique that allows reliable identification of the median nerve at several locations in the distal forearm and wrist by using the real-time capability of sonography. Median nerve pathologic characteristics are also described.

\section{Anatomy}

Accurate identification of the median nerve requires a thorough knowledge of its anatomic course and relationship to other structures in the distal forearm and wrist. The median nerve originates from 2 cervical nerve roots (the lateral cord $\mathrm{C} 5-\mathrm{C} 7$ and the medial cord C8 and $\mathrm{T} 1$ ) and travels down the arm and enters the forearm between the 2 heads of the pronator teres. The nerve passes distally in the volar aspect of the forearm between the flexor digitorum superficialis and the flexor digitorum profundus muscles.

Approximately $5 \mathrm{~cm}$ proximal to the flexor retinaculum, the median nerve courses around the radial or lateral edge of the flexor digitorum superficialis, where its position becomes more superficial. Just proximal to the carpus, the nerve lies between the tendons of the flexor digitorum superficialis and the flexor carpi radialis, partially deep to the tendon of palmaris longus (if it is present). The nerve then passes deep to the flexor retinaculum into the carpal tunnel of the wrist.

\section{Sonography}

A large peripheral nerve such as the median nerve shows alternating hypoechoic nerve bundles and hyperechoic connective tissue on sonography. ${ }^{3}$ Such nerves are typically surrounded by hyperechoic fat. On longitudinal imaging the echo texture of a peripheral nerve is coarser than that of a normal tendon. When imaged transversely, a peripheral nerve has a speckled appearance on sonography. 
Normal tendons appear hyperechoic with a fibrillar echo texture on sonography; however, a tendon will appear artifactually hypoechoic if not perpendicular to the ultrasound beam (also called anisotropy). ${ }^{4}$ In contrast, muscle is predominately hypoechoic with associated hyperechoic septations and fascial tissue.

\section{Technique}

Figure 1 illustrates the 3 levels at which static images were recorded in relation to the wrist. Figure 2 shows the position of the median nerve in relation to the flexor digitorum profundus and the flexor digitorum superficialis in the distal forearm in the axial plane corresponding to the levels illustrated in Figure 1.

It is important to use a high-frequency linear transducer, preferably $10 \mathrm{MHz}$ or higher, for optimal resolution. When scanning in the axial plane, at the level of the carpal tunnel, it is often difficult to differentiate hyperechoic tendons from the normal median nerve (Fig. 2A). However, when the transducer is moved proximally in the axial plane, several findings on sonography allow identification of the median nerve. First, the median nerve courses around the radial aspect of the flexor digitorum superficialis to ultimately lie between the flexor digitorum superficialis and the flexor digitorum profundus (Fig. 2B). Second, the hyperechoic tendons of the flexor digitorum superficialis and the flexor digitorum profundus are contiguous with hypoechoic muscle at the musculotendinous junctions, whereas the median nerve remains relatively hyperechoic. The hyperechoic fat surrounding the median nerve accentuates the contrast between the hyperechoic nerve and hypoechoic muscle proximally (Fig. 2C). These findings are most obvious on real-time sonography.

Figure. 1. Forearm of a 42-year-old man. Distal (A), middle (B), and proximal (C) levels shown correspond to the sonographic images described in Figure 2.

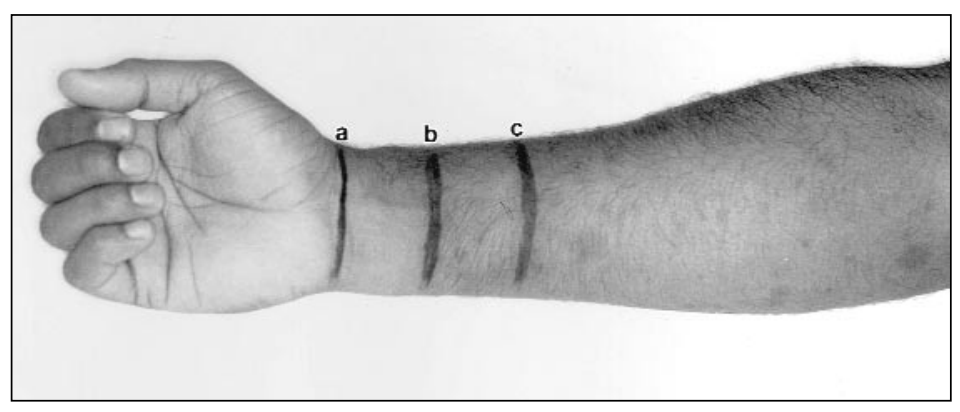

Figure 2. Axial sonograms of a wrist in a 42-year-old man. A, Corresponding to the distal level $(\mathrm{A})$ in Figure 1, the median nerve $(\mathrm{N})$ may be confused with the adjacent palmaris longus (curved arrow) and flexor carpi radialis (solid arrow). The median nerve lies superficial to tendons of the flexor digitorum (f). The radiocarpal ligament lies deep to flexor digitorum tendons (open arrow) and superficial to the ulna $(\mathrm{U})$ and radius (R). B, Corresponding to the middle level (B) in Figure 1, the median nerve $(\mathrm{N})$ courses around the radial aspect of the flexor digitorum superficialis $(\mathrm{S})$ lying between the flexor digitorum superficialis and the flexor digitorum profundus $(\mathrm{p})$. The pronator quadratus $(\mathrm{Q})$ lies deep to flexor muscles. C, Corresponding to the proximal level (C) in Figure 1, the median nerve $(\mathrm{N})$ now appears relatively hyperechoic, contrasting with adjacent hypoechoic muscle, and lies between the flexor digitorum superficialis (S) and the flexor digitorum profundus (P).
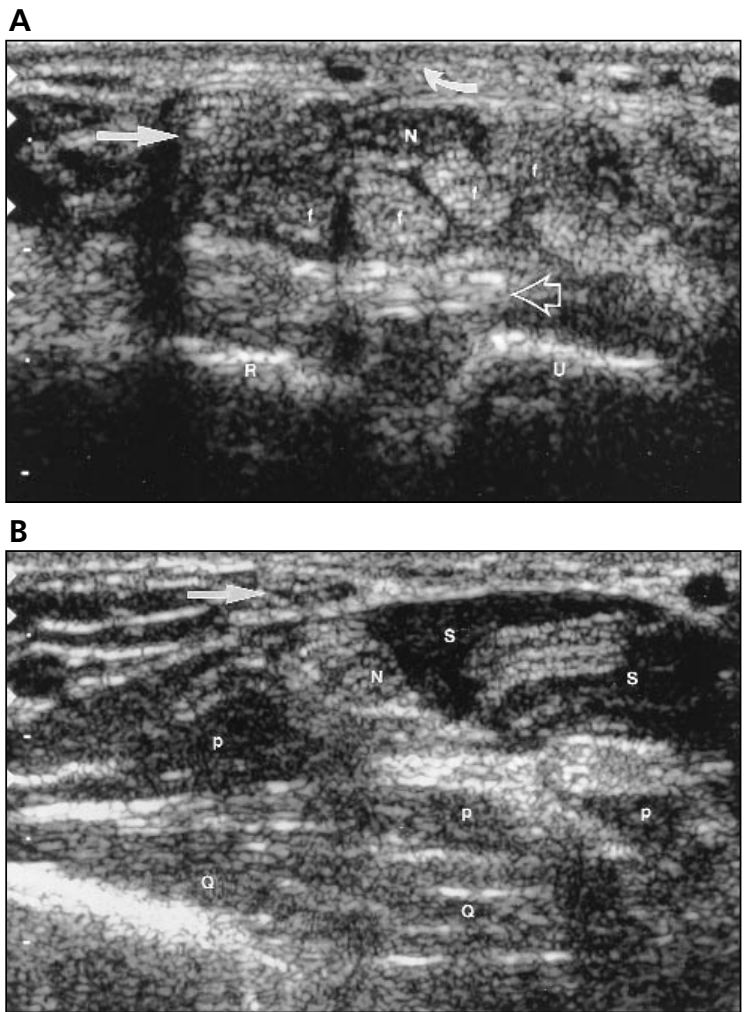

C

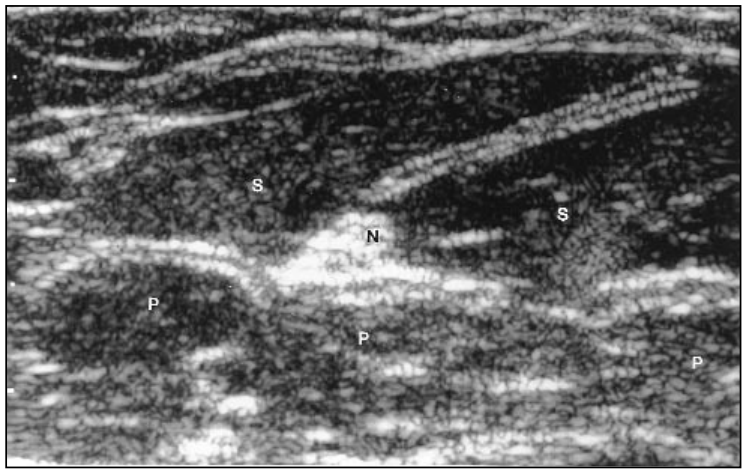


Once the median nerve is identified proximally, it can then be followed distally in the transverse-axial plane to the carpal tunnel. The echogenic surface of the pisiform at the ulnar aspect of the wrist defines the proximal carpal tunnel, the level at which the median nerve diameter is measured for carpal tunnel syndrome.

\section{Pathologic Characteristics}

Carpal tunnel syndrome is a compressive neuropathy of the median nerve in the carpal tunnel. Most cases are the result of activity that involves repetitive movement of the hand and wrist, but other associated conditions such as tenosynovitis, rheumatoid arthritis, and diabetes mellitus have been associated. Less commonly, carpal tunnel syndrome may be secondary to space-occupying lesions in the carpal tunnel itself.

Figure 3. Primary carpal tunnel syndrome. A, Longitudinal image of the median nerve $(\mathrm{N})$, which is in the expected anatomic location at the wrist but is of lower than expected echogenicity. This is emphasized by the echogenicity of the adjacent flexor tendons, which are immediately deep to it. $\mathrm{R}$ indicates radius; L, lunate; and $\mathrm{C}$, capitate. B, Cross-sectional image through the median nerve $(\mathrm{N})$, which allowed measurement of the cross-sectional area, which in this case was 15 $\mathrm{mm}^{2}$, consistent with mild to moderate electromyographic changes in carpal tunnel syndrome. ${ }^{5}$

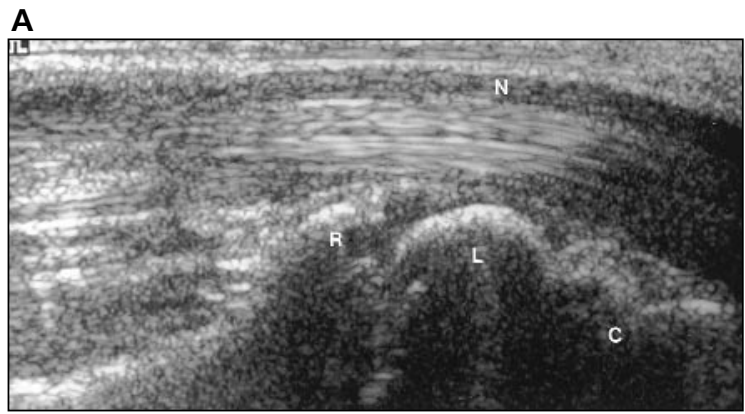

B

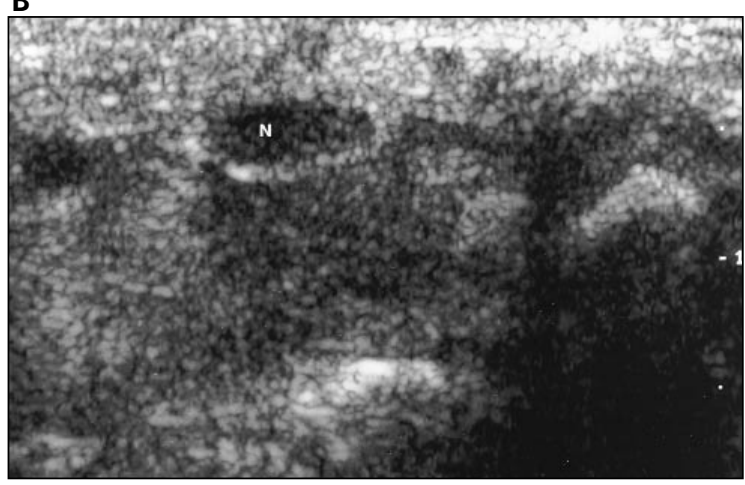

Sonographic findings in carpal tunnel syndrome include palmar bowing of the flexor retinaculum and hypoechoic enlargement of the median nerve. Nerve enlargement is assessed in the transverse plane just proximal to the carpal tunnel, where the median nerve is considered enlarged if the cross-sectional area is greater than $10 \mathrm{~mm}^{2}$ (transverse $\times$ anteroposterior dimensions) or greater than $9 \mathrm{~mm}^{2}$ (continuous boundary trace). ${ }^{2}$

In primary carpal tunnel syndrome (Fig. 3), sonography can be used to evaluate the median nerve by showing the cross-sectional area and the relative decreased echogenicity of the nerve because of edema. Secondary carpal tunnel syndrome may be due to space-occupying lesions such as a ganglion cyst (Fig. 4) or posttraumatic callus. The ability of sonography to show some of the secondary causes of carpal tunnel syndrome is one advantage sonography has over electromyographic studies in evaluating these patients. Sonography is also noninvasive and relatively painless.

Figure 4. Secondary carpal tunnel syndrome. The large anechoic mass (G; a surgically proven ganglion cyst) distorts the anatomy of the carpal tunnel. $\mathrm{N}$ indicates median nerve.

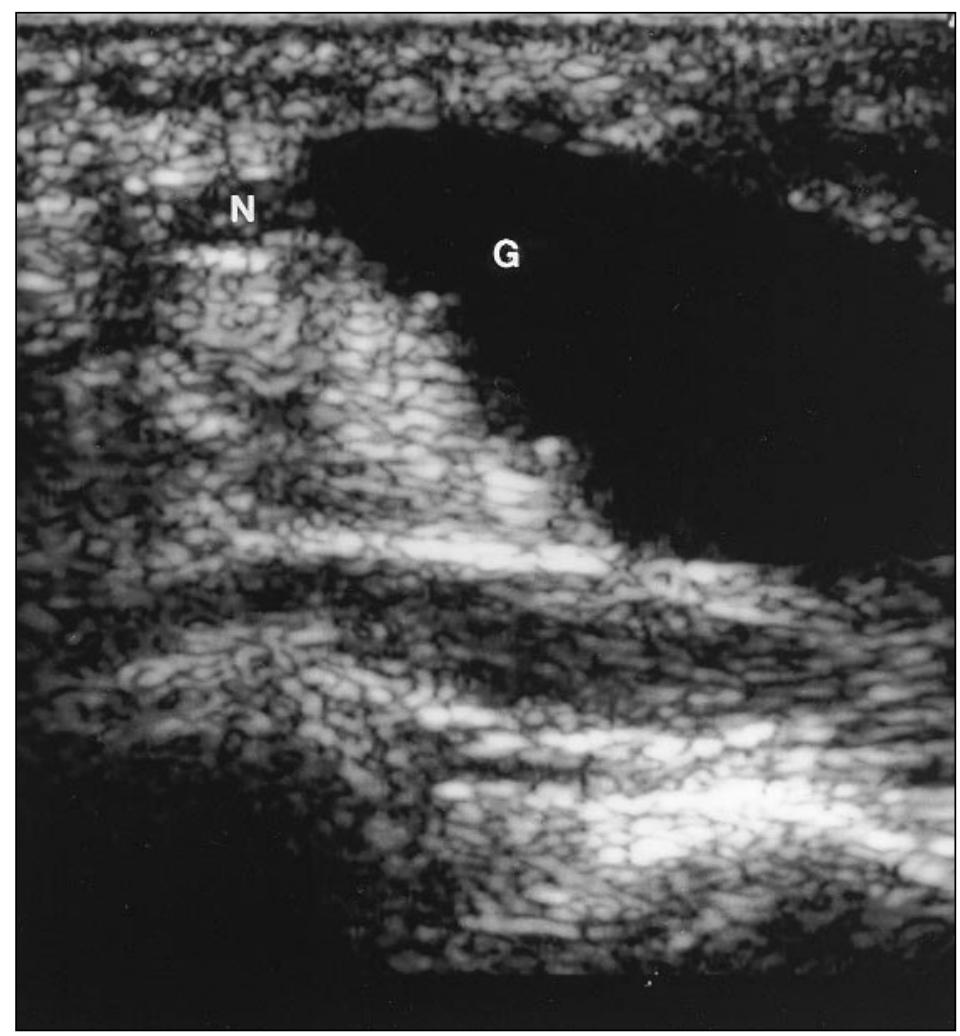




\section{Conclusion}

With knowledge of the sonographic anatomy of the wrist, this simple and effective technique allows accurate identification of the median nerve, facilitating evaluation of pathologic characteristics.

\section{References}

1. Jacobson JA, van Holsbeeck MT. Musculoskeletal ultrasonography. In: Boutin RD, Sartoris DJ (eds). Orthopedics Clinics of North America: Musculoskeletal Imaging Update. Part II. Vol 29. No 1. Philadelphia, PA: WB Saunders; 1998:135-167.

2. Duncan I, Sullivan P, Lomas F. Sonography in the diagnosis of carpal tunnel syndrome. AJR Am J Roentgenol 1999; 173:681-684.

3. Silvestri E, Martinoli C, Derchi LE, et al. Echotexture of peripheral nerves: correlation between US and histologic findings and criteria to differentiate tendons. Radiology 1995; 197:291-296.

4. Crass JR, van de Vegte GC, Harkavy LA. Tendon echogenicity: ex vivo study. Radiology 1988; 167 : 499-501.

5. Lee D, van Holsbeeck MT, Janevski PK, Ganos DL, Ditmars DM, Darian VB. Diagnosis of carpal tunnel syndrome: ultrasound versus electromyography. Radiol Clin North Am 1999; 37:859-872. 